\title{
MULTIPLE FACES OF PERSONALITY DOMAINS: REVALIDATING THE PROPOSED DOMAINS
}

\author{
Danilo Pesic $^{1}$, Dusica Lecic-Tosevski ${ }^{1,2,3}$, Marko Kalanj ${ }^{1}$, Olivera Vukovic ${ }^{1,2}$, \\ Marija Mitkovic-Voncina ${ }^{1,2}$, Amir Peljto ${ }^{1,2}$ \& Roger Mulder ${ }^{4}$ \\ ${ }^{I}$ Institute of Mental Health, Belgrade, Serbia \\ ${ }^{2}$ School of Medicine, University of Belgrade, Belgrade, Serbia \\ ${ }^{3}$ Serbian Academy of Sciences and Arts, Belgrade, Serbia \\ ${ }^{4}$ Department for Psychological Medicine, University of Otago, Christchurch, New Zealand
}

received: 26.3.2018;

revised: 11.12.2018;

accepted: 7.1.2019

\section{SUMMARY}

Background: Despite relatively consistent findings regarding the number of personality pathology domains, differences in domain structure remain. Recently the proposed ICD-11 domains were partially validated in a sample of patients with major depression producing five domains: Detached, Anankastic, Negative Emotional, Antisocial and Borderline. The aim of our study was to attempt to cross-validate these findings in a sample of patients primarily diagnosed with personality disorder (PD).

Subjects and methods: All subjects were assessed by Structured Clinical Interview for the DSM-IV Axis II PD. Exploratory factor analysis (EFA) was applied on fifty seven DSM PD symptoms selected to represent the five proposed domains.

Results: SCID II data were collected from a total of 223 subjects. The EFA extracted five factors. The first factor labeled as borderline-internalizing constituted of borderline together with avoidant and dependent items, the second, labeled as disinhibited/ borderline externalizing, incorporated narcissistic and histrionic items. The other three separate factors in our study labeled as antisocial, anankastic and detached, were less robust.

Conclusions: In our study five personality pathology domains were partly replicated. The most robust findings support the existence of the two factors, borderline-internalizing and disinhibited/borderline externalizing. However, the EFA was performed on a relatively low prevalence symptoms distribution, particularly for antisocial and schizoid factors.

Key words: personality disorders - personality pathology domain - ICD-11 proposed domains - factor analysis - borderline

\section{INTRODUCTION}

After thirty years of clinical work and research based on categorical diagnoses of personality disorders (PDs), the ICD-11 proposal for the classification of personality disorders has adopted a dimensional model (Tyrer et al. 2011), with advantages previously reported in a number of empirical data sets (Widiger \& Trull 2007). The proposed diagnostic procedure is stepped: establishing presence of personality disorder (PD), rating the severity level on a five-point scale, and an optional description of trait domains which describe the main features of personality pathology (Tyrer et al. 2011).

Severity of personality disturbance is a major component of assessment, and recent findings confirm that severity classification is a valuable construct (Kim et al. 2014).

Despite relatively consistent findings regarding the number of domains, the differences and heterogeneity of domain structure still remain. The five domains proposed by WHO ICD-11 Working Group are as follows: Negative Affective, Detachment, Dissocial, Disinhibition, and Anankastic (Tyrer et al. 2015). They were similar to four of five domains of the alternative DSM-5 system: negative emotional, detachment, antagonistic and disinhibition, respectively. The fifth domain in DSM-5 classification was labelled as psychotic (Oldham 2015).
In a recent study the proposed ICD-11 domains were partially validated in a large sample of patients with major depression (Mulder et al. 2016). The best fitting model identified also five domains which were labeled: Negative Emotional, Detached, Antisocial, Borderline, and Anankastic. The new Borderline domain incorporated borderline, histrionic, and narcissistic symptoms, while the Disinhibited domain was not a distinct domain but loaded onto Dissocial/Disinhibited factor.

The aim of our study was an attempt to cross-validate this model, in a sample of patients primarily diagnosed with personality disorders rather than mood disorders.

\section{SUBJECTS AND METHODS}

\section{Sample}

The study was carried out at the Institute of Mental Health, Belgrade from January 2011 to June 2016. The sample consisted of 223 inpatients diagnosed with PD according to ICD-10 criteria (World Health Organization 1992). Experienced clinicians confirmed the prior diagnosis for all participants.

The number of eligible patients who refused to participate in the study was $47(21.08 \%)$.

Out of 223 patients diagnosed with PD, 112 were recruited from day hospital for affective disorders and 
day hospital for adolescents (18 or more years of age), while 111 subjects were inpatients at the clinical ward of affective disorders. The purpose of their admission was to treat different mental disorders, primarily depression and anxiety disorders.

Exclusion criteria were: organic mental disorder, mental retardation, psychotic disorder, severe substance and drug abuse.

\section{Personality assessment}

All subjects were assessed by the Structured Clinical Interview for the DSM-IV Axis II Personality Disorders (SCID-II) (First et al. 1997). The assessment was performed when patients were in clinical remission of their comorbid state, at which point their subjective impression about their well-being and satisfaction with the level of symptom reduction matched that of the clinicians'. All subjects were also assessed by Structured Clinical Interview for the DSM-IV Axis I Disorders (SCID-I) (First et al. 2002).

The selection of PD symptoms to include in the analysis was guided by the framework laid out by Mulder and Tyrer (Mulder et al. 2016) since our aim was to cross-validate the factor structure of PD symptoms in a different sample. The same 57 DSM-IV symptom criteria were initially included.

In contrast to Mulder et al (2016) study, no symptoms with low base rate $(<5 \%)$ were found in our sample. Nevertheless, we still replicated the procedure of grouping the observed symptoms into a smaller number of item parcels, primarily to reduce data dimensionality, as well as to perform analysis on similarly structured data.

Our grouping procedure differed slightly from the original. Firstly, we classified borderline items into a smaller number of parcels (two), based on conceptual similarity: the first representing affective and impulsive instability (Bor4, Bor5, Bor6, Bor8), and the second (Bor1, Bor3 and Bor7 items) referring to identity problems. Secondly, we used conduct disorder symptoms instead of antisocial symptoms, which have previously been reported to predict future antisocial behavior (Mulder \& Joyce 1997).

\section{Statistics}

Exploratory factor analysis (EFA) was applied on fifty seven DSM PD symptoms selected to represent the five proposed domains. Principal axis factoring was used as the extraction method, being recommended as robust to violations of normality (Osborne \& Costello 2005), with Promax as the (oblique) rotation method. Analyses were conducted using IBM SPSS Statistics 19.

The study was conducted in line with the principles of the Ethics Research Code of the School of Medicine University of Belgrade. Informed consent was provided. The study was approved by the Ethical Committee of the Institute of Mental Health.

\section{RESULTS}

\section{Patient characteristics}

The SCID II data were collected from a total of 223 subjects (66.8\% female, $33.2 \%$ male), aged $18-67$ years (mean 37.6 \pm 13 ). Mean educational level was $12.58 \pm 2.59$ years: $11.1 \%$ of participants have completed only primary schooling, 58\% have completed secondary education, and $30.9 \%$ were university students, graduates or post-graduates.

Most prevalent ICD-10 diagnosis was Emotional Unstable PD, making up $65.1 \%$ of all diagnoses. The next most frequent diagnosis was Unspecified PD found in $9.7 \%$ of our sample. DSM-IV Axis I mental disorders were as follows: mood disorders $(58.2 \%)$, anxiety disorders $(46.4 \%)$ and substance-related disorders $(25 \%)$. Deliberate self-harm was registered among $43 \%$ of the total number of subjects, out of which $17 \%$ were suicide attempts.

\section{SCID II Personality Assessment}

Descriptive statistics (symptom means, SDs, medians, percent of cases with scores above threshold for diagnosis) and measures of reliability, internal consistency (Cronbach's $\alpha$ ) and inter-rater agreement (Cohen's $\kappa$ ) for SCID-II scales are shown in Table 1.

Table 1. Descriptive statistics and measures of reliability for SCID-II scales

\begin{tabular}{lcccccc}
\hline & $\begin{array}{c}\text { Symptoms } \\
\text { (mean) }\end{array}$ & $\begin{array}{c}\text { Symptoms } \\
(\text { SD) }\end{array}$ & $\begin{array}{c}\text { Symptoms } \\
\text { (median) }\end{array}$ & $\begin{array}{c}\text { \% above } \\
\text { threshold }\end{array}$ & Cronbach's $\alpha$ & Kappa \\
\hline Avoidant & 2.34 & 2.14 & 2.00 & 26.9 & 0.77 & 0.71 \\
Dependent & 2.85 & 2.27 & 3.00 & 26.9 & 0.64 & 0.72 \\
Obsessive-compulsive & 3.79 & 1.85 & 4.00 & 55.6 & 0.55 & 0.66 \\
Borderline & 4.76 & 2.87 & 5.00 & 57.4 & 0.81 & 0.86 \\
Histrionic & 1.88 & 1.75 & 2.00 & 9.0 & 0.64 & 0.75 \\
Narcissistic & 3.47 & 2.32 & 3.00 & 33.6 & 0.68 & 0.72 \\
Conduct & 0.95 & 1.87 & 0.00 & 13.5 & 0.80 & 0.91 \\
Paranoid & 2.71 & 1.93 & 2.00 & 33.6 & 0.66 & 0.63 \\
Schizoid & 1.99 & 1.51 & 2.00 & 18.8 & 0.53 & 0.60 \\
Schizotypal & 2.02 & 1.44 & 2.00 & 8.1 & 0.62 & 0.18 \\
\hline
\end{tabular}


Table 2. Prevalence rates of analyzed symptoms

\begin{tabular}{|c|c|c|}
\hline Symptom & DSM PD Criterion & Prevalence $(\%)$ \\
\hline Frantic attempts to... & Borderline 1 (Bor1) & 51.6 \\
\hline Identity disturbance... & Borderline 3 (Bor3) & 59.6 \\
\hline Impulsivity in at least... & Borderline 4 (Bor4) & 52.0 \\
\hline Recurrent suicidal behaviour... & Borderline 5 (Bor5) & 47.1 \\
\hline Affective instability... & Borderline 6 (Bor6) & 56.1 \\
\hline Chronic feelings of emptiness... & Borderline 7 (Bor7) & 64.6 \\
\hline Inappropriate intense anger... & Borderline 8 (Bor8) & 55.2 \\
\hline Avoids occupational activities... & Avoidant 1 (Av1) & 30.0 \\
\hline Unwilling to get involved with people... & Avoidant 2 (Av2) & 26.9 \\
\hline Shows restraint within intimate relationships... & Avoidant 3 (Av3) & 29.6 \\
\hline Is preoccupied with being criticised... & Avoidant 4 (Av4) & 48.4 \\
\hline Is inhibited... & Avoidant 5 (Av5) & 33.2 \\
\hline Views self as socially inept... & Avoidant 6 (Av6) & 34.5 \\
\hline Unusually reluctant to take risks... & Avoidant 7 (Av7) & 32.3 \\
\hline Has difficulty making decisions... & Dependent 1 (Dep1) & 17.5 \\
\hline Has difficulty expressing disagreement... & Dependent 3 (Dep3) & 32.7 \\
\hline Goes to excessive lengths... & Dependent 5 (Dep5) & 47.5 \\
\hline Feels uncomfortable... & Dependent 6 (Dep6) & 48.4 \\
\hline Urgently seeks another relationship... & Dependent 7 (Dep7) & 22.0 \\
\hline Is unrealistically preoccupied... & Dependent 8 (Dep8) & 41.3 \\
\hline Perceives attacks on his or her character... & Paranoid 6 (Par6) & 46.2 \\
\hline Grandiose sense of self-importance... & Narcissistic 1 (Nar1) & 47.1 \\
\hline Preoccupied with fantasies of unlimited success... & Narcissistic 2 (Nar2) & 40.8 \\
\hline Has a sense of entitlement... & Narcissistic 5 (Nar5) & 50.2 \\
\hline Is interpersonally exploitative... & Narcissistic 6 (Nar6) & 30.0 \\
\hline Lacks empathy... & Narcissistic 7 (Nar7) & 28.7 \\
\hline Is uncomfortable in situations... & Histrionic 1 (His 1$)$ & 33.2 \\
\hline Interactions with others is.... & Histrionic 2 (His2) & 32.7 \\
\hline Displays rapidly shifting and shallow... & Histrionic 3 (His3) & 17.0 \\
\hline Constantly uses physical appearance... & Histrionic 4 (His4) & 28.3 \\
\hline Is suggestible... & Histrionic 7 (His7) & 26.9 \\
\hline Before the age of $15 \ldots$ bullied other kids... & Conduct 1 (Con 1$)$ & 9.0 \\
\hline ...started fights... & Conduct 2 (Con 2 ) & 12.1 \\
\hline ...hurt or threatened someone with a weapon... & Conduct 3 (Con 3 ) & 2.7 \\
\hline ...deliberately tortured someone... & Conduct 4 (Con4) & 5.8 \\
\hline ...tortured or hurt animals... & Conduct 5 (Con5) & 4.0 \\
\hline ...robed... muged... & Conduct 6 (Con6) & 1.8 \\
\hline ...forced someone to have sex... & Conduct 7 (Con7) & 1.3 \\
\hline$\ldots$...set fires... & Conduct 8 (Con 8$)$ & 3.1 \\
\hline ...deliberately destroyed things... & Conduct 9 (Con9) & 5.4 \\
\hline ...broke into houses... & Conduct $10($ Con 10$)$ & 1.8 \\
\hline ...lied a lot or "conned" other people & Conduct 11 (Con11) & 10.8 \\
\hline ...ran away from home... & Conduct 13 (Con13) & 10.8 \\
\hline Before the age of thirteen. stayed out very late... & Conduct 14 (Con14) & 10.3 \\
\hline Before the age of thirteen...skipped school... & Conduct 15 (Con15) & 4.5 \\
\hline Preoccupied with details... & Obsessive compulsive $1(\mathrm{Oc} 1)$ & 56.5 \\
\hline Shows perfectionism... & Obsessive compulsive 2 (Oc2) & 49.3 \\
\hline Is excessively devoted to work... & Obsessive compulsive 3 (Oc3) & 25.6 \\
\hline Is over-conscientious... & Obsessive compulsive 4 (Oc4) & 62.3 \\
\hline Is unable to discard... & Obsessive compulsive 5 (Oc5) & 50.7 \\
\hline Is reluctant to delegate... & Obsessive compulsive 6 (Oc6) & 48.0 \\
\hline Adopts a miserly... & Obsessive compulsive 7 (Oc7) & 15.7 \\
\hline Shows rigidity... & Obsessive compulsive 8 (Oc8) & 71.7 \\
\hline Does not enjoy close relationships... & Schizoid 1 (Szo1) & 24.7 \\
\hline Almost always chooses solitary activities... & Schizoid 2 (Szo2) & 39.9 \\
\hline Little interest in sexual experiences... & Schizoid 3 (Szo3) & 35.4 \\
\hline Takes pleasure in few, if any... & Schizoid 4 (Szo4) & 49.8 \\
\hline Lacks close friends... & Schizoid 5 (Szo5) & 30.9 \\
\hline Appears indifferent to praise or criticism... & Schizoid 6 (Szo6) & 18.8 \\
\hline
\end{tabular}


There was a significant overlap between SCID-II PD diagnoses. A total of $64.1 \%$ of patients fulfilled SCID-II criteria for two or more PDs, with $92.2 \%$ of patients diagnosed as Borderline PD fulfilling the criteria for at least one additional PD.

Internal consistency was good for Borderline and Conduct disorder scales, and in the acceptable range for the Avoidant scale, while other scales displayed modest (Dependent, Histrionic, Narcissistic, Paranoid, and Schizotypal) or poor (Obsessive-compulsive and Schizoid) internal consistency. Inter-rater agreement was moderate to strong for most symptoms and scales. However, a significant problem was noted during SCIDII personality assessment. There was low agreement between raters for schizotypal and schizoid symptoms. Almost all schizotypal symptoms had very low $(<0.2)$ inter-rater reliability, with particularly low rater agreement in symptoms based on interviewer's observations. The same problem occurred with schizoid symptom 7 ("shows emotional coldness"). Therefore, we decided to exclude this schizoid item and all schizotypal items from further analysis, retaining the same number of slightly different symptoms. Prevalence rates of analyzed symptoms are shown in Table 2. Symptom parcels descriptive statistics and inter-correlations are shown in Table 3.

\section{Factor analysis}

EFA was performed on 21 item parcels, providing a ratio of 10.6 cases per variable. Kaiser-Meyer-Olkin measure of sampling adequacy was 0.81 , and Bartlett's test of sphericity was significant $\left(\chi^{2}(210)=1350.62\right.$, $\mathrm{p}<0.01$ ), indicating that data were suitable for factor analysis. Five factors with eigenvalues over 1 were extracted, accounting for a total of $41.39 \%$ of the variance. Unique factor loadings and communalities for variables included in the analysis are shown in Table 4.

The first factor accounted for $21.29 \%$ of variance, and consisted of avoidant, dependent and borderline symptom parcels, as well the Paranoid 6 symptom ("sensitivity to criticism and rejection by others"). Factor loadings were high (greater than 0.5) for avoidant and dependent symptoms, as well as borderline symptoms which described internalizing behaviours (frantic efforts to avoid abandonment, identity disturbance and chronic feeling of emptiness), and somewhat smaller (but still above 0.3 ) for the externalizing symptoms of impulsivity, emotional instability and anger. Since it included both internalizing and borderline dimensions, we labeled this factor "borderlineinternalizing".

The second factor accounted for $10.20 \%$ of variance, and consisted of histrionic and narcissistic symptoms (with histrionic items loading more strongly). Avoidant symptoms associated with social isolation also had a negative cross loading on this factor. Given that this factor largely included disinhibited and externalizing features, we labeled this factor "disinhibited/borderline externalizing".

Table 4. Unique factor loadings and communalities for PD symptom parcels

\begin{tabular}{|c|c|c|c|c|c|c|}
\hline Symptom parcel & $\begin{array}{l}\text { Borderline- } \\
\text { Internalizing }\end{array}$ & $\begin{array}{l}\text { Disinhibited/ } \\
\text { Borderline } \\
\text { Externalizing }\end{array}$ & Antisocial & Anankastic & Detached & $\begin{array}{c}\text { Commu- } \\
\text { nalities }\end{array}$ \\
\hline (17) Av4 Av7 & 0.877 & & & & -0.415 & 0.673 \\
\hline (19) Av3 Av5 Av6 & 0.798 & & & & & 0.555 \\
\hline (18) Av1 Av2 & 0.665 & -0.319 & & & & 0.525 \\
\hline (21) Dep1 Dep3 Dep5 & 0.636 & & & & & 0.304 \\
\hline (5) Bor1 Bor3 Bor7 & 0.552 & & & & & 0.559 \\
\hline (20) Dep6 Dep7 Dep8 & 0.524 & & & & & 0.335 \\
\hline (6) Bor4 Bor5 Bor6 Bor8 & 0.366 & & & & & 0.511 \\
\hline (11) Par6 & 0.334 & & & & & 0.340 \\
\hline (1) His1 His4 His7 & & 0.905 & & & & 0.654 \\
\hline (2) His2 His3 & & 0.666 & & & & 0.457 \\
\hline (3) Nar1 Nar2 & & 0.496 & & & & 0.332 \\
\hline (4) Nar5 Nar6 Nar7 & & 0.385 & & & & 0.301 \\
\hline (7) Con 1 Con 2 Con 6 Con 9 Con 10 & & & 0.862 & & & 0.737 \\
\hline (8) Con3 Con4 Con 5 & & & 0.666 & & & 0.385 \\
\hline (10) Con 11 Con 12 & & & 0.541 & & & 0.352 \\
\hline (9) Con 13 Con 14 Con 15 & & & 0.428 & & & 0.246 \\
\hline (14) Oc1 Oc2 & & & & 0.663 & & 0.337 \\
\hline (15) Oc3 Oc4 Oc5 & & & & 0.563 & & 0.275 \\
\hline (16) Oc6 Oc7 Oc8 & & & & 0.398 & & 0.418 \\
\hline (13) Szo5 Szo6 & & & & & 0.515 & 0.235 \\
\hline (12) Szo1 Szo2 Szo3 Szo4 & & & & & 0.350 & 0.376 \\
\hline
\end{tabular}

Note: factor loadings $<0.2$ are suppressed; Abbreviations for DSM PD symptom criteria: His - Histrionic; Nar - Narcissistic; Bor - Borderline; Con - Conduct; Par - Paranoid; Szo - Schizoid; Oc - Obsessive-compulsive; Av - Avoidant; Dep - Dependent. 
Table 5. Factor inter-correlations

\begin{tabular}{|c|c|c|c|c|c|}
\hline Factor & $\begin{array}{l}\text { Borderline- } \\
\text { Internalizing }\end{array}$ & $\begin{array}{c}\text { Disinhibited/Borderline } \\
\text { Externalizing }\end{array}$ & Antisocial & Anankastic & Detached \\
\hline Borderline-Internalizing & 1.000 & & & & \\
\hline Disinibited/Borderline Externalizing & 0.279 & 1.000 & & & \\
\hline Antisocial & 0.232 & 0.394 & 1.000 & & \\
\hline Anankastic & 0.642 & 0.274 & 0.229 & 1.000 & 0.410 \\
\hline Detached & 0.461 & 0.333 & 0.336 & 0.410 & 1.000 \\
\hline
\end{tabular}

$\mathrm{p}<0.01$ for all correlations

Conduct disorder symptoms loaded clearly and, in general strongly, onto the third factor, labeled "antisocial", explaining $5.64 \%$ of the variance.

Obsessive-compulsive symptoms constituted a fourth factor (labeled "anankastic"), but it accounted for a relatively small portion of the variance $(2.76 \%)$.

The fifth factor (labeled "detached") consisted of schizoid symptoms, plus negatively cross-loaded avoidant symptoms 4 and 7, implying "lack of concern for reactions of others". This factor also explained a very small portion of the variance $(2.5 \%)$. The correlation of extracted factors is shown in Table 5.

A strong correlation between borderline-internalizing and anankastic factors was observed, while other correlations were of a moderate size. Factor score estimates for the five factors were created from an unweighted sum of the individual items contained in the item parcels loading on each factor and factor reliabilities estimated using Cronbach's alpha. Estimated reliabilities were good for the borderline-internalizing $(\alpha=0.86)$ and antisocial $(\alpha=0.80)$ factors, acceptable for the disinhibited/borderline externalizing factor $(\alpha=0.71)$, and poor for the anankastic $(\alpha=0.55)$ and detached $(\alpha=0.53)$ factors.

\section{DISCUSSION}

In our study we partially replicated the five domain structures. The most robust findings supported the existence of the first two factors labeled as borderlineinternalizing and disinhibited/borderline externalizing factor. The other three separate factors in our study labeled as antisocial, anankastic and detached, were significantly less robust in comparison with the first two factors.

The Negative Affective factor in the Mulder et al (2016) study included avoidant and dependent items, with only one borderline item. In contrast, in our study this factor incorporated all borderline items in addition to avoidant and dependent items. A strong connection between DSM IV anxious cluster, PDs and BPD has already been reported in previous studies (Zanarini et al. 1998). Several studies have classified both avoidant and dependent traits as a part of broader internalizing factor (Mulder et al. 2011), which sometimes included emotional dysregulation, so we chose to label this factor as "borderline-internalizing".
The second factor in the Mulder et al (2016) study was labeled as Borderline. It included the majority of borderline and all narcissistic and histrionic items with the addition of paranoid 6 item ("sensitivity to criticism and rejection by others"). In our study, the second factor incorporated only narcissistic and histrionic items, while paranoid 6 item was united with avoidant and dependent items in the borderline-internalizing factor. Taking into account numerous studies which report histrionic and narcissistic traits (alongside borderline traits) as a part of disinhibited, or as a part of broad externalizing dimension (Mulder et al. 2011) we chose to label our second factor as disinhibited/borderline externalizing factor.

The internalizing/externalizing model has proven to be useful for characterizing Axis I disorders in DSM-IV classification and could be linked to and translated to personality structure (James \& Taylor 2008, Krueger et al. 2001). Moreover, studies have reported that the covariation between internalizing and externalizing dimensions may be clearer after examining the connection between personality and these features and that internalizing and externalizing factors both contribute to BPD (Hudson et al. 2014). Additional findings showed that neuroticism as a personality trait is an inherited marker of both internalizing and externalizing pathologies (Hink et al. 2013).

The remaining three domains were found to be very similar to those in the study of Mulder et al (2016) so we labeled them dissocial, detached, and anankastic. Despite relatively small portion of variance accounted for by the antisocial factor (around 5\%) which could be due to the low prevalence of Antisocial PD in our sample (1.5\%) - conduct disorder clearly constituted a separate factor, with satisfactory estimated factor reliability, which was moderately correlated to the externalizing factor.

Low reliability of schizotypal items in our sample was a particular problem, but weak evidence of a detached factor was found, based only on schizoid items.

Obsessive-compulsive symptoms loaded clearly onto the anankastic factor. However, it accounted for a low portion of variance (in contrast to prevalence of obsessive-compulsive symptoms in our sample), had poor estimated reliability, and was strongly correlated with the "borderline-internalizing" factor. High prevalence of obsessive symptoms in our sample could be 
due to high rate of Emotionally Unstable PD and high level of Axis II comorbidity, especially with the anxious PDs cluster, already reported in the literature (Zanarini et al. 1998). Comorbid obsessive-compulsive PD is associated with severe forms of BPD (Nordahl \& Nysaeter 2005, Palomares et al. 2016).

Our findings showed a considerable frequency of multiple PD diagnoses, with most common co-occurrence of BPD with other PD syndromes. Similarly with our study, Barachina et al (2011) found that approximately $74 \%$ of patients with BPD were noted to have at least one co-occurring Axis II disorder. In their sample the most common were paranoid, avoidant, dependent, and passive-aggressive PDs (Barrachina et al. 2011). Comparable results in the study of Zanarini et al (1998) showed that PDs from all three clusters were more frequent among BPD, especially anxious and odd.

\section{Limitations}

Considering the size of our sample we performed factor analysis on a relatively low prevalence symptoms distribution for some PDs (conduct and schizoid symptoms). We did not perform objective assessment of mood and anxious disorder, therefore influence of state on personality traits was conducted only by clinical assessment. We applied only EFA, and did not test the five-factor model using confirmatory factor analysis (CFA). The recommendation in literature is to perform both an EFA on half of the sample, and a CFA on the other half when cross-validating results of factor studies (Brown 2006). We decided to apply only EFA with regard to limitations imposed by number of participants, since splitting our sample in half would reduce number of cases per variable ratio.

Our sample consisted of patients with personality disorders and acute exacerbation of symptoms of AxisI disorders requiring intensive treatment (hospital or day hospital treatment), with the predominance of depression and anxiety, with a considerable prevalence of self-harmers, and with the exclusion of acute psychosis, at the institution that treats the patients on voluntary basis only (no involuntary hospitalizations). This may have implications in terms of the PD sample not being representative but rather a sample of more severe and low functioning PDs with considerable psychiatric symptoms. Furthermore, considering that patients in the sample are treated on voluntary basis, the low representation of antisocial personality disorder is understandable, given that aggressive antisocial behavior is often associated with low cooperativeness and refusal of voluntary treatment. Finally, excluding the patients with acute psychotic disorder could have been the reason for missing those personality disorders that are most likely to seek treatment in psychotic decompensations, such as schizotypal personality disorder, possibly explaining the low prevalence of this disorder in our sample.

\section{CONCLUSIONS}

In our study we partially replicated the five domain structures from the Mulder et al. (2016) study. Our findings support the presence of two factors labeled as borderline-internalizing and disinhibited/borderline externalizing factor. Borderline symptoms together with avoidant and dependent symptoms constituted one factor, which we labeled borderline internalizing factor. The second factor incorporated narcissistic and histrionic symptoms which we have labeled the disinhibited/borderline externalizing factor.

There are fundamentally different views about keeping the term "borderline" in personality disorder nosology (Bateman 2011, Tyrer 2009). We chose to keep the term in order to emphasize how common the cooccurrence of BPD symptoms with other PD diagnoses is in most of the studies. It seems that BPD persistently stays on the borderline between dimensional and categorical (Trull et al. 2011), personality structure and the level of functioning.

More focus is needed on this type of research in a sample of child and adolescent population and on developmental aspect of personality disorders.

\section{Acknowledgements:}

This study was supported by the Ministry of Science, Republic of Serbia (project no. ON175013).

\section{Conflict of interest:}

Dusica Lecic Tosevski and Roger Mulder are members of the WHO Working Group for ICD-11 Personality Disorders Classification.

\section{Contribution of individual authors:}

Danilo Pesic: study design, literature review, data collection, first draft.

Dusica Lecic-Tosevski: study design, literature review, approval of the final version.

Marko Kalanj: study design, statistical analysis, first draft.

Olivera Vukovic: literature review, first draft.

Marija Mitkovic-Voncina: first draft, data collection, statistical analysis.

Amir Peljto: literature review, data collection.

Roger Mulder: approval of the final version.

\section{References}

1. Barrachina J, Pascual JC, Ferrer M, Soler J, Rufat MJ, Andión $O$ et al: Axis II comorbidity in borderline personality disorder is influenced by sex, age, and clinical severity. Compr Psychiatry 2011; 52:725-730

2. Bateman AW: Throwing the baby out with the bathwater? Personal Ment Health 2011; 5:274-280 
3. Brown TA: Confirmatory factor analysis for applied research. Methodology in the social sciences. New York, NY: Guilford Press, 2006

4. First MB, Gibbon $M$, Spitzer RL, Williams JBW \& Benjamin LS: Structured Clinical Interview for DSM-IV Axis II Personality Disorders (SCID-II). Washington, D.C.: American Psychiatric Press, Inc, 1997

5. First MB, Spitzer RL, Gibbon $M \&$ Williams JBW: Structured clinical interview for DSM-IV-TR Axis I Disorders, Research Version, Patient Edition. (SCID-I/P). New York: Biometrics Research, New York State Psychiatric Institute, 2002

6. Hink LK, Rhee SH, Corley RP, Cosgrove VE, Hewitt JK, Schulz-Heik RJ et al: Personality dimensions as common and broadband-specific features for internalizing and externalizing disorders. J Abnorm Child Psychol 2013; 41:939-957

7. Hudson JI, Zanarini MC, Mitchell KS, Choi-Kain LW \& Gunderson JG: The contribution of familial internalizing and externalizing liability factors to borderline personality disorder. Psychol Med 2014; 44:2397-2407

8. James LM \& Taylor J: Revisiting the structure of mental disorders: borderline personality disorder and the internalizing/ externalizing spectra. Br J Clin Psychol 2008; 47:361-380

9. Kim YR, Blashfield R, Tyrer P, Hwang ST \& Lee HS: Field trial of a putative research algorithm for diagnosing ICD-11 personality disorders in psychiatric patients: 1 . Severity of personality disturbance. Personal Ment Health 2014; 8:67-78

10. Krueger $R F, M c G u e ~ M \&$ Iacono $W G$ : The higher-order structure of common DSM mental disorders: Internalization, externalization, and their connections to personality. Pers Individ Dif 2001; 30:1245-1259

11. Mulder RT, Horwood J, Tyrer P, Carter J \& Joyce PR: Validating the proposed ICD-11 domains. Personal Ment Health 2016; 10:84-95

12. Mulder RT \& Joyce PR: Temperament and the structure of personality disorder symptoms. Psychol Med 1997; 27:99-106

13. Mulder RT, Newton-Howes G, Crawford MJ \& Tyrer PJ: The central domains of personality pathology in psychiatric patients. J Pers Disord 2011; 25:364-377
14. Nysaeter TE \& Nordahl HM: Comorbidity of borderline personality disorder with other personality disorders in psychiatric outpatients: how does it look at 2-year followup? Nord J Psychiatry 2012; 36:209-214

15. Oldham JM: The alternative DSM-5 model for personality disorders. World Psychiatry 2015; 14:234-236

16. Osborne JW \& Costello AB: Best Practices in Exploratory Factor Analysis: Four Recommendations for Getting the Most From Your Analysis. Practical Assessment, Research \& Evaluation/PARE 2005; 10:1-9

17. Palomares N, McMaster A, Díaz-Marsá M, de la Vega I, Montes A, \& Carrasco JL: Multiple cluster axis II comorbidity and functional outcome in severe patients with borderline personality disorder. Actas Esp Psiquiatr 2016; 44:212-221

18. Trull TJ, Distel MA \& Carpenter RW: DSM-5 borderline personality disorder: At the border between a dimensional and a categorical view. Curr Psychiatry Rep 2011; 13:43-49

19. Tyrer P: Why borderline personality disorder is neither borderline nor a personality disorder. Personal Ment Health 2009; 3:86-95

20. Tyrer P, Crawford $M \&$ Mulder $R$ (on behalf of the ICD11 working group on personality disorders): Reclassifying personality disorders. Lancet 2011; 377:1814-1815

21. Tyrer P, Crawford M, Mulder R, Blashfield R, Farnam A, Fossati $A$ et al: The rationale for the reclassification of personality disorders in the 11th revision of the International Classification of Diseases (ICD-11). Personal Ment Health 2011; 5:246-259

22. Tyrer P, Reed GM \& Crawford MJ: Classification, assessment, prevalence, and effect of personality disorder. Lancet 2015; 385:717-726

23. Widiger TA \& Trull TJ: Plate tectonics in the classification of personality disorder: shifting to a dimensional model. Am Psychol 2007; 62:71-83

24. World Health Organization. The ICD-10 Classification of Mental and Behavioural Disorders, 1992

25. Zanarini MC, Frankenburg FR, Dubo ED, Sickel AE, Trikha A, Levin A et al: Axis II comorbidity of borderline personality disorder. Compr Psychiatry 1998; 39:296302

Correspondence:

Prof. Dusica Lecic-Tosevski, MD, PhD

Institute of Mental Health

Palmoticeva 37, 11000 Belgrade, Serbia

E-mail: dusica.lecictosevski@gmail.com 